\title{
Abundances of planetary nebulae NGC 40 and NGC $6153^{\star}$
}

\author{
S. R. Pottasch ${ }^{1}$, J. Bernard-Salas ${ }^{1,2}$, D. A. Beintema ${ }^{1,2}$, and W. A Feibelman ${ }^{3}$ \\ 1 Kapteyn Astronomical Institute, PO Box 800, 9700 AV Groningen, The Netherlands \\ 2 SRON Laboratory for Space Research, PO Box 800, 9700 AV Groningen, The Netherlands \\ 3 Laboratory for Astronomy and Solar Physics, Code 681, Goddard Space Flight Center, MD, USA
}

Received 20 May 2003 / Accepted 24 July 2003

\begin{abstract}
The ISO and IUE spectra of the elliptical nebulae NGC 40 and NGC 6153 are presented. These spectra are combined with the spectra in the visual wavelength region to obtain a complete, extinction corrected, spectrum. The chemical composition of the nebulae is then calculated and compared to previous determinations. A discussion is given of: (1) the recombination line abundances, (2) the exciting stars of the nebulae, and (3) possible evolutionary effects.
\end{abstract}

Key words. ISM: abundances - planetary nebulae: individual: NGC 40, NGC 6153 - infrared: ISM - ISM: lines and bands

\section{Introduction}

NGC 40 and NGC 6153 are similar nebulae in several respects. They are both large nebulae, elliptical in shape and somewhat open on the long end. Brighter structures are seen within the nebulae which could be interpreted as a torus-like structure. NGC 6153 has a size of about $18^{\prime \prime} \times 28^{\prime \prime}$ for the brighter regions, while NGC 40 is somewhat larger, about $38^{\prime \prime} \times 38^{\prime \prime}$. Both nebulae have faint emission which extends beyond these dimensions. A good photograph of NGC 40 can be found in Meaburn et al. (1996); one of NGC 6153 can be found in Liu et al. (2000).

Another similarity is the $6 \mathrm{~cm}$ radio flux density, as well as the far infrared flux. Because there is more extinction in the direction of NGC 6153, this nebula appears weaker in the visible, and it is much harder to see in the ultraviolet.

Both of these nebulae have been studied before. NGC 6153 had been poorly studied before the launch of the IRAS satellite which showed that it had a strong infrared emission line spectrum. This stimulated the work of Pottasch et al. (1986) showing the very high abundances in this nebula. There is a recent, very detailed and very interesting work by Liu et al. (2000) which contains both careful measurements of the visual spectrum, and the ISO LWS (but not the SWS) measurements as well. This work showed that the abundances of important elements obtained from recombination lines give a different (and higher) abundance than those obtained from collisionally

Send offprint requests to: S. R. Pottasch,

e-mail: pottasch@astro.rug.nl

* Based on observations with ISO, an ESA project with instruments funded by ESA Member States (especially the PI countries: France, Germany, The Netherlands and the UK) and with the participation of ISAS and NASA. excited lines. The reason for this is at present not satisfactorally explained.

NGC 40 has been studied earlier, probably because it is a northern hemisphere object. Unfortunately, there are no visual spectra covering the entire nebula. The best spectrum is that of Clegg et al. (1983) which covers a bright region of approximately $12^{\prime \prime} \times 7^{\prime \prime}$. There is also a spectrum taken by Aller \& Czyzak (1979) which refers to a bright part of the nebula, probably the same region. Ultraviolet (IUE) spectra of the same region of the nebula, as well as spectra of other regions of the nebula (Feibelman 1999) have been made. The size of the IUE diaphragm is not large enough to cover the entire nebula, so that in this case, as well as in NGC 6153, a correction for the diaphragm size must be made.

The ISO diaphragm is only able to measure the entire nebula in the long wavelength region. The ISO LWS spectra (which cover a spectral region from $45 \mu \mathrm{m}$ until almost $200 \mu \mathrm{m})$, are taken with a diaphragm large enough to cover the entire nebula. The ISO SWS spectra, covering the spectral region from $2.4 \mu \mathrm{m}$ to about $45 \mu \mathrm{m}$, are taken with a smaller diaphragm so that only part of the nebula is measured.

The exciting star of NGC 40 was classified as a Wolf-Rayet WC8 star by Smith \& Aller (1969) and it has remained in this class in the most recent classification by Crowther et al. (1998). It has an observed magnitude $V=11.55$ (Ciardullo et al. 1999). The star is also classified as $\mathrm{O}$ VI since it shows weak emission lines of this ion at $\lambda 3811 \AA$ and $\lambda 3834 \AA$. The stellar temperature is usually given as approximately $T=35000 \mathrm{~K}$, which is consistent with the low ionization of the nebula. Bianchi \& Grewing (1987) give a temperature of $T=90000 \mathrm{~K}$ based on the rather uncertain ultraviolet continuum. We return to this point in Sect. 6. The exciting star of NGC 6153 is considerably fainter. Ciardullo et al. (1999) give an observed magnitude 
$V=15.55$. Because the extinction in the direction of this nebula is larger than for NGC 40 the actual difference is only about a factor of 10 . The stellar temperature, judging from the ionization state of the nebula, is considerably higher. The spectrum of the central star has not yet been carefully studied. According to Liu et al. (2000) this star also shows the $\lambda 3811 \AA$ line of O VI. It does not show any other stellar emission lines, except possibly the C IV at $\lambda 5801 \AA$ and $5812 \AA$. This would place it in the group of weak emission line stars which are sometimes called low gravity PG1159 stars. These stars appear to be similar in composition to Wolf-Rayet stars in the sense that their atmospheres are hydrogen free and consist mainly of helium and carbon in about equal amounts.

The purpose of this paper is to obtain more accurate abundances for these nebula using the ISO spectra. The reasons for this have been discussed in earlier papers (e.g. see Pottasch \& Beintema 1999; Pottasch et al. 2000, 2001; Bernard Salas et al. 2001), and can be summarized as follows.

The most important advantage is that the infrared lines originate from very low energy levels and thus give an abundance which is not sensitive to the temperature in the nebula, nor to possible temperature fluctuations. Furthermore, when a line originating from a high-lying energy level in the same ion is observed, it is possible to determine an effective temperature at which the lines in that particular ion are formed. When the effective temperature for many ions can be determined, it is possible to make a plot of effective temperature against ionization potential, which can be used to determine the effective temperature for ions for which only lines originating from a high energy level are observed. Use of an effective electron temperature takes into account the fact that ions are formed in different regions of the nebula. At the same time possible temperature fluctuations are taken into account.

Use of the ISO spectra have further advantages. One of them is that the number of observed ions used in the abundance analysis is approximately doubled, which removes the need for using large "Ionization Correction Factors", thus substantially lowering the uncertainty in the abundance. A further advantage is that the extinction in the infrared is almost negligible, eliminating the need to include large correction factors.

This paper is structured as follows. First the spectrum of NGC 40 is discussed (in Sect. 2). Section 3 discusses the spectrum of NGC 6153. This prepares the way to discussing the chemical composition of both nebulae in Sect. 4, and comparing the resultant abundances with those made earlier (in Sect. 5). In Sect. 5.2 the relation between nebular abundances determined from collisionally excited lines and from recombination lines is considered. In Sect. 6 the central stars are considered in more detail. Finally Sect. 7 gives a comparison with the abundances determined in other PN, and a general discussion.

\section{The spectrum of NGC 40}

\subsection{ISO observations}

Most of the ISO SWS observations were made with the SWSO2 observing template which gives good spectral resolution for a limited number of lines (TDT 44401917, 81101203, 81101304,
$81803104,82701607,85303501)$. This was supplemented by an SWS01 observation (TDT 30003803). The intensity of the lines found in the spectrum is shown in Table 1 . The uncertainty of the stronger lines is less than $10 \%$. The measurements were centered at $\mathrm{RA}(2000) 00^{\mathrm{h}} 13^{\mathrm{m}} 01.0^{\mathrm{s}}$ and $\operatorname{Dec}(2000)$ $+72^{\circ} 31^{\prime} 18.9^{\prime \prime}$, which is very close to the center of the nebula. Because the diaphragm used was $14^{\prime \prime} \times 20^{\prime \prime}$ below $12 \mu \mathrm{m}$ and somewhat larger above this wavelength, only a part of the nebula was measured by the SWS. Therefore a factor must be applied to correct for the missing flux. Because of the different diaphragm sizes, this factor depends on the size of the diaphragm and thus depends somewhat on the wavelength. The factor was found in the following way. For the range below $12 \mu \mathrm{m}$ the factor was determined so that the observed hydrogen lines, especially the $\operatorname{Br} \alpha$ and $\operatorname{Br} \beta$ lines agree with the total $\mathrm{H} \beta$ which in turn is derived from the total radio emission to be discussed below. The correction factor for this wavelength region is 5.5. The correction for the wavelength region between $12 \mu \mathrm{m}$ and $27 \mu \mathrm{m}$ is found by comparison with the IRAS measurements made with a very large diaphragm so that the entire nebula was measured. The IRAS measurement of the $12.8 \mu \mathrm{m}$ [Ne II] given by Pottasch et al. (1986) is $1.5 \times 10^{-10} \mathrm{erg} \mathrm{cm}^{-2} \mathrm{~s}^{-1}$. We have rereduced the IRAS measurements and obtained almost the same result: $1.46 \times 10^{-10} \mathrm{erg} \mathrm{cm}^{-2} \mathrm{~s}^{-1}$. This leads to a correction factor of 4.3. The continuum spectrum between $13 \mu \mathrm{m}$ and $20 \mu \mathrm{m}$ of the IRAS was a factor of 4.0 higher than the ISO flux in this wavelength region; this is the factor which has been used. Finally, the flux in the spectral region between $30 \mu \mathrm{m}$ and $46 \mu \mathrm{m}$ was corrected so that it agreed with the LWS measurements at $46 \mu \mathrm{m}$ which contain the entire nebula. This requires a correction factor of 2.5. The results are given in Table 1. The intensities are given for the entire nebula (after the correction factors have been applied). The LWS measurements are reported and discussed by Liu et al. (2001).

\subsection{Extinction}

There are several methods for obtaining the extinction: (1) comparison of radio emission with $\mathrm{H} \beta$ flux, (2) comparison of observed and theoretical Balmer decrement, (3) dip at $\lambda 2200 \AA$, (4) photometry of the exciting star. First, however, we will discuss the radio emission and the $\mathrm{H} \beta$ flux.

\subsubsection{The $6 \mathrm{~cm}$ radio emission}

Several measurements of the $6 \mathrm{~cm}$ flux density have been made. The most reliable appears to be that of George et al. (1974) who have made a map of the nebula with the WRST telescope, thus avoiding nearby sources. They give a flux density of $457 \mathrm{mJy}$. The Green Bank single dish also measured the nebula at this wavelength, giving a somewhat higher value: 587 mJy (Becker et al. 1991, rereduced by Gregory \& Condon 1991, who find $506 \mathrm{mJy}$ ). The danger of confusion is real, if only because NGC 40 lies on the rim of the supernova remnant CTA1. Incidentally Seiber et al. (1979) found that the radio emission of CTA1 is completely depolarised at the position of NGC 40 and concluded that it lies in front of CTA1, which has a 
Table 1. ISO observations of NGC 40 (in units of $10^{-13} \mathrm{erg} \mathrm{cm}^{-2} \mathrm{~s}^{-1}$ ).

\begin{tabular}{|c|c|c|c|}
\hline Ident. & $\overline{\lambda(\mu \mathrm{m})}$ & Intens.(1) & Intens.(2) \\
\hline H I 6-4 & 2.626 & 12 & 66 \\
\hline Н I $10-5$ & 3.039 & 1.5 & 8.2 \\
\hline Н I 9-5 & 3.297 & 2 & 11 \\
\hline H I & 4.052 & 25 & 137 \\
\hline [Ar II] & 6.984 & 55 & 301 \\
\hline [Ar III] & 8.990 & 30 & 164 \\
\hline$[\mathrm{Ne}$ II $]$ & 12.811 & 340 & 1360 \\
\hline [Ne III] & 15.554 & 5 & 20 \\
\hline [P III] & 17.888 & 9 & 36 \\
\hline [S III] & 18.711 & 148 & 592 \\
\hline [Fe III] & 22.926 & 4 & 16 \\
\hline [O IV] & 25.890 & $\leq 0.6$ & $\leq 2.4$ \\
\hline$[\mathrm{Fe} \mathrm{II}]$ & 25.988 & 1.0: & 4.0: \\
\hline [S III $]$ & 33.476 & 180 & 450 \\
\hline [Si II] & 34.810 & 28 & \\
\hline$[\mathrm{O} \mathrm{IIII}]^{*}$ & 52 & 200 & 200 \\
\hline$[\mathrm{N} \mathrm{IIII}]^{*}$ & 57 & 170 & 170 \\
\hline$[\mathrm{OI}]^{*}$ & 63 & 221 & 221 \\
\hline$[\mathrm{O} \text { III }]^{*}$ & 88 & 119 & 119 \\
\hline$[\mathrm{N} \mathrm{II}]^{*}$ & 122 & 32 & 32 \\
\hline$[\mathrm{O} \mathrm{I}]^{*}$ & 146 & 7.5 & 7.5 \\
\hline$[\mathrm{C} \mathrm{II}]^{*}$ & 157 & 203 & 203 \\
\hline
\end{tabular}

Intens.(1) are measured values; Intens.(2) are intensities corrected for the diaphragm size (see Sect. 2.1).

* These data are from Liu et al. (2001).

distance of $1.5 \mathrm{kpc}$. The $21 \mathrm{~cm}$ flux density is $510 \mathrm{mJy}$ (Condon \& Kaplan 1998) made with the VLA D-array. If the nebula is optically thin at $21 \mathrm{~cm}$ this would correspond to a $6 \mathrm{~cm}$ flux density of $450 \mathrm{mJy}$, which agrees with the value of George et al. (1974). We shall use $457 \mathrm{mJy}$ in the present analysis.

\subsubsection{The $\mathrm{H} \beta$ flux}

Two measurements of the $\mathrm{H} \beta$ flux are given in the literature. Carrasco et al. (1983) give $\log \mathrm{H} \beta=-10.37$, while Liller (1955) finds $\log \mathrm{H} \beta=-10.64$. The value given by Carrasco et al. (1983) seems to be the better one, not only because it is the newer one and it is part of extensive measurements which appear trustworthy, but also because it is more consistent with the measurement of the [S III] line at $\lambda 9532 \AA$ by Hippelein $\&$ Munch (1981). These authors measure the total intensity of this line over the entire nebula; it is only consistent with the other lines, both in the infrared as well as in the visible, when the higher $\mathrm{H} \beta$ flux is used.

\subsubsection{The value of the extinction $E_{B-V}$}

- Using the above value of the $6 \mathrm{~cm}$ radio flux density and values of electron temperature and helium abundance which will presently be discussed, we obtain the intrinsic

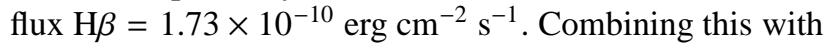
the measured value $\mathrm{H} \beta=4.28 \times 10^{-11} \mathrm{erg} \mathrm{cm}^{-2} \mathrm{~s}^{-1}$ gives a value of extinction $C=0.605$ or $E_{B-V}=0.41$.
- The extinction determined from the Balmer decrement differs somewhat according to the author. Aller \& Czyzak (1979) give $C=0.65$, Clegg et al. (1983) find $C=0.70$ and Sabbadin et al. (2000) give $C=0.60$. Earlier measurements, e.g. Aller et al. (1972) gave much lower values which are not included in the average: $C=0.65$ $E_{B-V}=0.44$.

- The extinction determined from the dip at $\lambda 2200 \AA$ is somewhat more difficult because the flux is weak near the center of the dip. Pottasch et al. (1977) give $E_{B-V}=0.38$, using filter photometry on the ANS satellite. Bianchi \& Grewing (1987) find $E_{B-V}=0.50$ from IUE measurements.

- Narrow band photometry has been carried out by Crowthers et al. (1998), who find $E_{B-V}=0.42$.

These values are quite similar. In the remainder of this paper we use the value $E_{B-V}=0.41$ or $C=0.60$, together with the extinction curve of Fluks et al. (1994).

\subsection{The visual spectrum}

The visual spectrum has been measured by Clegg et al. (1983), Aller \& Czyzak (1979) and Aller et al. (1972). The measurements of Clegg et al. (1983) refer to the brightest patch of luminosity located about $14^{\prime \prime}$ northwest of the exciting star, and are integrated over a region of $8^{\prime \prime} \times 12^{\prime \prime}$. Those of Aller \& Czyzak are taken with a 2 " $\times 2$ " diaphragm and "correspond to a low excitation region". Finally the measurements of Aller et al. (1972) are averaged over several positions in the nebula, and are considered by the authors as "appropriate to the nebula as a whole". These latter measurements are more uncertain than the others; especially the low intensity values are so uncertain that they have not been shown. The first five columns of Table 2 give the measured intensities relative to $\mathrm{H} \beta=100$ for those lines which are of interest. In the last column the unreddened intensities are given, normalized to the total flux: $\mathrm{H} \beta=1.73 \times 10^{-10} \mathrm{erg} \mathrm{cm}^{-2} \mathrm{~s}^{-1}$. An average value of the individual measurements, scaled to the total flux, is used. It is not clear how close these values approach the actual integrated nebular fluxes of the entire nebula.

\subsection{The IUE ultraviolet spectrum}

Many IUE observations of this nebula exist. Most of them are centered at the position of the central star which then dominates the spectrum. Several observations have been made of the nebula far enough from the central star so that no stellar light is measured. Several of the low dispersion spectra are made in roughly the same region as the visual measurements of Clegg et al. (1983). These measurements are reported by Clegg et al. (1983) and have been rereduced by Feibelman (1999) using the NEWSIPS reduction program. These measurements are given in the third column of Table 3 (I(line)). They can be supplemented by high resolution measurements which include the central star. The stellar spectrum can be separated from the nebular spectrum because the stellar line emission is much broader.

Because these nebular observations only contain a part of the nebula, a correction must be made for the diaphragm size; 
Table 2. Visual spectrum of NGC 40 (unreddened intensity in units of $10^{-12} \mathrm{erg} \mathrm{cm}^{-2} \mathrm{~s}^{-1}$ ).

\begin{tabular}{|c|c|c|c|c|c|}
\hline \multirow{2}{*}{$\begin{array}{c}\lambda \\
(\AA)\end{array}$} & \multirow[t]{2}{*}{ Ion } & \multicolumn{3}{|c|}{ Intensities } & \multirow{2}{*}{$\begin{array}{l}\text { Unred. } \\
\text { intens. }\end{array}$} \\
\hline & & (1) & $(2)$ & (3) & \\
\hline 3727 & [O II] & 264 & 264 & 253 & 657 \\
\hline 3869 & [Ne III] & 0.25 & & & 0.59 \\
\hline 4068 & [S II] & & 3.0 & 2.3 & 6.8 \\
\hline 4076 & [S II] & & 0.91 & 0.8 & 2.1 \\
\hline 4102 & $\mathrm{H} \delta$ & 19 & 19 & 23 & 42.4 \\
\hline 4267 & C II & 0.35 & & & 0.72 \\
\hline 4340 & $\mathrm{H} \gamma$ & 39 & & 43 & 81.0 \\
\hline 4363 & [O III] & 0.13: & & & $0.27:$ \\
\hline 4471 & He I & 1.9 & 2.1 & 3.2 & 4.2 \\
\hline 4861 & $\mathrm{H} \beta$ & 100 & 100 & 100 & 173 \\
\hline 5007 & [O III $]$ & 19 & 12 & 29 & 43 \\
\hline 5518 & [Cl II] & $0.25:$ & 0.26 & & $0.35:$ \\
\hline 5538 & {$[\mathrm{Cl} \mathrm{II}]$} & 0.35: & 0.26 & & 0.47 : \\
\hline 5755 & {$[\mathrm{~N}$ II $]$} & 2.7 & 3.1 & & 3.7 \\
\hline 5876 & He I & 9.0 & 9.1 & & 11.3 \\
\hline 6312 & [S III] & 0.74 & 0.72 & & 0.86 \\
\hline 6563 & $\mathrm{H} \alpha$ & 480 & 500 & & 520 \\
\hline 6584 & {$[\mathrm{~N}$ II $]$} & 450 & 460 & & 490 \\
\hline 6717 & [S II] & 15.4 & 18 & & 18 \\
\hline 6731 & [S II] & 23.2 & 22.5 & & 25 \\
\hline 7136 & [Ar III] & 6.9 & 8.9 & & 8.0 \\
\hline 7320 & [O II] & 11.5 & & & 11.4 \\
\hline 7330 & [O II] & 9.7 & & & 9.4 \\
\hline 9531 & [S III] & & & & $227^{*}$ \\
\hline
\end{tabular}

(1) Clegg et al. (1983), (2) Aller \& Czyzak (1979), (3) Aller et al. (1972).

The (:) indicates uncertain values.

* The [S III] $19531 \AA$ intensity was taken from Hippelein \& Munch (1981).

alternatively use can be made of the continuum measurement in the same diaphragm for which the line was measured, to obtain the ratio of the line to $\mathrm{H} \beta$. This latter method, which was also used by Clegg et al. (1983), has the advantage that the ratio obtained in this way is independent of the extinction. In detail the method is as follows. The theoretical ratio $I$ (continuum) $/ I(\mathrm{H} \beta)$ is computed, where $I$ (continuum) consists of two quantum emission, Balmer continuum and a small contribution from neutral helium emission. The ratio is independent of electron density because all the continua and the $\mathrm{H} \beta$ mission have the same density dependence; it has only a small dependence on the electron temperature since all the emission sources change in much the same way. A value of $T_{\mathrm{e}}=10^{4} \mathrm{~K}$ was used. It was assumed that the two quantum emission is produced by all recombinations to the 2 s level, and that recombinations to the $2 \mathrm{p}$ level produce Ly $\alpha$ photons which are removed from the nebula. The observed $I$ (continuum) is shown in the fourth column of Table 3 . The value of $I($ line $)$ is then multiplied by the theoretical ratio $I$ (continuum) $/ I(\mathrm{H} \beta)$ and divided by the observed $I$ (continuum) to give the ratio $I($ line $) / I(\mathrm{H} \beta)$, shown in the fifth column. This ratio, independent of extinction, has been directly used for determining ionic abundance (it is assumed that the ratio is the same over the entire nebula).
Table 3. IUE spectrum of NGC 40.

\begin{tabular}{ccccccc}
\hline \hline \multirow{2}{*}{$\begin{array}{c}\lambda \\
(\AA)\end{array}$} & Ion & \multicolumn{2}{c}{ Intensities } & & \multicolumn{2}{c}{ Ratio } \\
\cline { 7 - 7 } \cline { 6 - 7 } & & $(1)$ & $(2)$ & & $(3)$ & $(4)$ \\
\hline 1550 & {$[$ C IV] } & 5.13 & 1.69 & & 0.246 & 0.268 \\
1663 & O III] & $\leq 0.7$ & 1.54 & & $\leq 0.033$ & $\leq 0.034$ \\
1762 & C II & 1.35 & 1.34 & & 0.101 & 0.061 \\
1883 & Si III] & 0.45 & 1.01 & & 0.026 & 0.022 \\
1909 & C III] & 11.2 & 0.87 & & 0.72 & 0.564 \\
2325 & C II] & 10.1 & 0.49 & & 0.873 & 0.636 \\
2470 & [O II] & 2.31 & 0.67 & & 0.142 & 0.094 \\
2740 & C II & 0.59 & 1.14 & & 0.021 & 0.015 \\
2841 & C II & 1.36 & 1.26 & & 0.049 & 0.031 \\
\hline
\end{tabular}

(1) $I($ line $)$ - all intensity units $10^{-13} \mathrm{erg} \mathrm{cm}^{-2} \mathrm{~s}^{-1}$;

(2) $I$ (continuum) - all intensity units $10^{-14} \mathrm{erg} \mathrm{cm}^{-2} \mathrm{~s}^{-1}$;

(3) $I($ line $) / I(\mathrm{H} \beta)$ from continuum measurement;

(4) $I($ line $) / I(\mathrm{H} \beta)$ from $[\mathrm{O} \mathrm{II}]$ ratio.

There is another way of correcting for the diaphragm size of the IUE aperture. This is by relating the IUE intensities to the $\mathrm{H} \beta$ intensity in the visual by means of the [O II] line ratio $I(\lambda 2470) / I(\lambda 7325)$. These lines arise from the same levels, and have a theoretical ratio of 0.78 for all electron densities and temperatures of interest. The extinction is of importance in determining the observational ratio, and a value of $E_{B-V}=0.41$ has been used. In addition the assumption has been made that the ratio of these lines to $\mathrm{H} \beta$ remains constant over the nebula. This results in the determination that the IUE diaphragm covers $24 \%$ of the nebula, and this leads to the $I($ line $) / I(\mathrm{H} \beta)$ ratios given in the last column of Table 3 . This correction for the diaphragm size is very similar to that used for the SWS spectrum between $12 \mu \mathrm{m}$ and $27 \mu \mathrm{m}$ The agreement with the ratio obtained from the measurement of the continuum is reasonably good, although there seems to be a wavelength dependence. This is not caused by a wrong choice of the extinction $E_{B-V}$ since any other reasonable value would not improve the situation. But the cause is not clear; there could be a number of possible origins. First, the measured continuum could be inaccurate; it is weak in this region. Secondly, the assumed extinction in this region could be wrong, either because the average extinction law used (Fluks et al. 1994) is wrong, or because the dust in this direction has somewhat different properties than the average dust. Thirdly, the calculation of the theoretical continuum could be wrong for a reason yet unknown. We shall use the average of the two methods. This introduces a maximum error of $25 \%$.

\section{The spectrum of NGC 6153}

\subsection{ISO observations}

Two ISO SWS01 observations were made of this nebula. We have used only one of them, that taken on 09 Feb. 1996 (TDT 08402713). The reason for this is that this spectrum appears less noisy and more consistent than the other observation. The position measured for both spectra was the same: $\operatorname{RA}(2000) 16^{\mathrm{h}} 31^{\mathrm{m}} 30.9^{\mathrm{s}}$ and $\operatorname{Dec}(2000)$ $-40^{\circ} 15^{\prime} 22.4^{\prime \prime}$. This was supplemented by an SWS02 measurement (TDT 28901214) on which only three lines could 
be measured. Liu informs us that the position measured is not the center of the nebula but $3.49^{\prime \prime}$ east and $9.0^{\prime \prime}$ south of the center. In addition the uncertainty of the observed position is $3^{\prime \prime}$. The diaphragm was not large enough to cover the entire nebula and a correction factor is necessary. Because this nebula is smaller than NGC 40, the correction factor is lower. For the range below $12 \mu \mathrm{m}$ the factor was determined so that the observed hydrogen lines, especially the $\operatorname{Br} \alpha$ and $\operatorname{Br} \beta$ lines agree with the total $\mathrm{H} \beta$ which in turn is derived from the total radio emission which will be discussed below. The correction factor for this wavelength region is 2.3. The correction for the wavelength region between $12 \mu \mathrm{m}$ and $27 \mu \mathrm{m}$ is found by comparison with the IRAS measurements made with a very large diaphragm so that the entire nebula was measured. The IRAS measurement of the $15.5 \mu \mathrm{m}$ [Ne III] given by Pottasch et al. (1986) is $7.5 \times 10^{-10} \mathrm{erg} \mathrm{cm}^{-2} \mathrm{~s}^{-1}$. We have rereduced the IRAS measurements and obtained a somewhat larger result: $11.1 \times 10^{-10} \mathrm{erg} \mathrm{cm}^{-2} \mathrm{~s}^{-1}$. Liu et al. (2000) have also rereduced the IRAS data and obtained a smaller value: $5.4 \times 10^{-10} \mathrm{erg} \mathrm{cm}^{-2} \mathrm{~s}^{-1}$ This leads to a very uncertain correction factor between 1.7 and 3.4. Because the diaphragm in this wavelength range is only slightly larger than that in the lower wavelength range and the additional flux may be quite small, we have used the value of 2.0 between $12 \mu \mathrm{m}$ and $27 \mu \mathrm{m}$. It is possible that some ions are more concentrated than others, so that the correction factor may depend somewhat on the ion discussed. This increases the uncertainty of the derived intensities.

Finally, the flux in the spectral region between $30 \mu \mathrm{m}$ and $46 \mu \mathrm{m}$ was corrected so that it agrees at $46 \mu \mathrm{m}$ with the LWS measurements which contain the entire nebula. This requires a correction factor of 1.25 . The results are given in Table 4. The intensities are given for the entire nebula (after the correction factors have been applied). All the measurements have been corrected for extinction (see below), but the correction is small and only important at the lower wavelengths. The LWS measurements are reported and discussed by Liu et al. (2000, 2001).

\subsection{Extinction}

The extinction can be determined from a comparison of the radio emission with the $\mathrm{H} \beta$ flux. The $6 \mathrm{~cm}$ flux density is given by Griffith et al. (1994) as $663 \mathrm{mJy}$, and is similar to an earlier value cited by Cahn et al. (1992): $632 \mathrm{mJy}$. Gregory et al. (1994) give $641 \mathrm{mJy}$. We will use $650 \mathrm{mJy}$, which together with the helium abundance and electron temperature given below, lead to an intrinsic $\mathrm{H} \beta=2.15 \times 10^{-10} \mathrm{erg} \mathrm{cm}^{-2} \mathrm{~s}^{-1}$. Combining this with the observed $\log \mathrm{H} \beta=-10.86$ (Cahn et al. 1992), a value of $C=1.19$ or $E_{B-V}=0.81$ is obtained.

The Balmer decrement given by Liu et al. (2000) gives a similar answer. The $\mathrm{H} \alpha / \mathrm{H} \beta$ ratio gives $E_{B-V}=0.90$ and the $\mathrm{H} \gamma / \mathrm{H} \beta$ and $\mathrm{H} \delta / \mathrm{H} \beta$ ratios give 0.80 and 0.82 respectively. In the remainder of this paper a value $E_{B-V}=0.81$ will be used.

\subsection{The visual spectrum}

The visual spectrum has been determined by several authors. The best spectrum to date is that of Liu et al. (2000) who have
Table 4. ISO observations of NGC6153 (in units of $10^{-13} \mathrm{erg} \mathrm{cm}^{-2} \mathrm{~s}^{-1}$ ).

\begin{tabular}{|c|c|c|c|}
\hline Ident. & $\lambda(\mu \mathrm{m})$ & Intens.(1) & Intens.(2) \\
\hline$\overline{\mathrm{HI} \mathrm{Br} \beta}$ & 2.625 & 55.2 & 127 \\
\hline He II 7-6 & 3.091 & 8.5 & 19 \\
\hline Н г Вr $\alpha$ & 4.053 & 78.3 & 180 \\
\hline [Ar II] & 6.983 & 106 & 244 \\
\hline [Na III] & 7.321 & 16.5 & 38 \\
\hline [Ar III] & 8.994 & 499 & 1148 \\
\hline [S IV] & 10.511 & 2490 & 5730 \\
\hline [Cl IV] & 11.756 & 38: & 87: \\
\hline [Ne II] & 12.818 & 124 & 248 \\
\hline [Ne III] & 15.556 & 3340 & 6680 \\
\hline [S III] & 18.712 & 928 & 1856 \\
\hline [Ar II] & 21.836 & 15: & 30: \\
\hline [Fe III] & 22.94 & 4.5 & 9 \\
\hline [O IV] & 25.901 & 1046 & 2090 \\
\hline [S III] & 33.487 & 413 & 516 \\
\hline [Si II] & 34.815 & 48.4 & 60.5 \\
\hline [Ne III] & 36.012 & 666 & 832 \\
\hline [O III $]^{*}$ & 52 & 5400 & 5400 \\
\hline$[\mathrm{N} \text { III }]^{*}$ & 57 & 1550 & 1500 \\
\hline$[\mathrm{O} \mathrm{III}]^{*}$ & 88 & 1580 & 1580 \\
\hline$[\mathrm{N} \mathrm{II}]^{*}$ & 122 & $\leq 4$ & $\leq 4$ \\
\hline
\end{tabular}

Intens.(1) are measured values; Intens.(2) are intensities corrected for the diaphragm size (see text).

* These data are from Liu et al. (2001).

measured the entire nebula by uniformly scanning a long slit across the whole object. From their detailed measurements, we have reproduced in Table 5 those line intensities which are important for the present work. The third column of the table gives the observed intensities relative to $\mathrm{H} \beta=100$ and the fourth column gives the total intensity corrected for extinction.

\subsection{The ultraviolet IUE spectrum}

Even though NGC 6153 is a bright radio source, it is difficult to measure in the ultraviolet because of the rather large extinction. Only eight IUE spectra have been taken, four by the shortwavelength camera and four by the long wavelength camera. Two were not well centered and will be ignored in the present discussion. All are low dispersion spectra and quite noisy. A further difficuly is that the spectra contain both radiation from the central star and the nebula which are difficult to separate.

The spectra are in NEWSIPS format that yields better $\mathrm{S} / \mathrm{N}$ than the old IUESIPS. In the earlier work (Liu et al. 2000; Pottasch et al. 1986) only three lines were detected in the SWP spectra, in spite of the fact that Liu et al. (2000) also coadded the spectra. This indicates that the weaker line intensities should be taken with caution. All measureable emission features, both of the nebula and the star, are listed in Table 6. The 1984 and 1986 data were coadded and exposure weighted, but the errors are considerable for the weaker lines. The wavelength and identification are given in the first two columns of the table, the third column gives the measured flux, the fourth column is the flux after correction for the extinction, and the last column gives the ratio of the line emission to $\mathrm{H} \beta$. 
Table 5. Visual spectrum of NGC 6153 (unreddened total intensity in units of $10^{-12} \mathrm{erg} \mathrm{cm}^{-2} \mathrm{~s}^{-1}$ ).

\begin{tabular}{|c|c|c|c|}
\hline $\begin{array}{c}\lambda \\
(\AA)\end{array}$ & Ion & $\begin{array}{l}\text { Relative } \\
\text { intensity }\end{array}$ & $\begin{array}{c}\text { Total }^{*} \\
\text { intensity }\end{array}$ \\
\hline 3726 & {$[\mathrm{O} \text { II }]^{\dagger}$} & 8.82 & 40.9 \\
\hline 3729 & {$[\mathrm{O} \mathrm{II}]^{\dagger}$} & 4.73 & 21.1 \\
\hline 3869 & {$[\mathrm{Ne} \mathrm{III}]$} & 46.8 & 194. \\
\hline 4068 & [S II] & 0.58 & 2.09 \\
\hline 4076 & [S II] & 0.20 & 0.71 \\
\hline 4102 & $\mathrm{H} \delta$ & 16.1 & 56.8 \\
\hline 4267 & C II & 1.56 & 5.03 \\
\hline 4340 & $\mathrm{H} \gamma$ & 33.3 & 100.3 \\
\hline 4363 & [O III] & 2.90 & 8.85 \\
\hline 4471 & He I & 4.84 & 14.0 \\
\hline 4686 & He II & 11.2 & 27.5 \\
\hline 4711 & [Ar IV] & 2.26 & 5.44 \\
\hline 4740 & [Ar IV] & 2.14 & 5.05 \\
\hline 4861 & $\mathrm{H} \beta$ & 100 & 215 \\
\hline 5007 & [O III] & 987 & 1952. \\
\hline 5191 & [Ar III] & 0.125 & 0.22 \\
\hline 5518 & [Cl II $]$ & 0.865 & 1.25 \\
\hline 5538 & {$[\mathrm{Cl} \mathrm{II}]$} & 1.11 & 1.60 \\
\hline 5755 & {$[\mathrm{~N}$ II $]$} & 1.47 & 1.88 \\
\hline 5876 & $\mathrm{He} \mathrm{I}$ & 35.6 & 42.1 \\
\hline 6312 & [S III] & 3.09 & 3.05 \\
\hline 6563 & $\mathrm{H} \alpha$ & 772 & 705. \\
\hline 6584 & {$[\mathrm{~N}$ II $]$} & 127. & 115. \\
\hline 6717 & [S II] & 8.74 & 7.55 \\
\hline 6731 & [S II] & 14.6 & 12.6 \\
\hline 7136 & [Ar III] & 61.9 & 47.1 \\
\hline 7320 & [O II] & 6.08 & 4.28 \\
\hline 7330 & {$[\mathrm{O} \mathrm{II}]$} & 5.47 & 3.81 \\
\hline
\end{tabular}

$\dagger$ Taken from measurements on the minor axis.

${ }^{*}$ The total fluxes are extinction corrected.
Table 6. IUE spectrum of NGC 6153.

\begin{tabular}{|c|c|c|c|c|}
\hline \multirow{2}{*}{$\begin{array}{c}\lambda \\
(\AA)\end{array}$} & \multirow[t]{2}{*}{ Ion } & \multicolumn{2}{|c|}{ Intensities } & \multirow{2}{*}{$\begin{array}{c}\text { Ratio } \\
\text { (3) }\end{array}$} \\
\hline & & (1) & (2) & \\
\hline 1240 & $\mathrm{NV}$ & 1.6 & 23.0 & 0.30 \\
\hline 1248 & C III & 1.2 & 17.0 & 0.22 \\
\hline 1293 & O VI & 2.3 & 23.0 & 0.30 \\
\hline 1334 & C II & 1.7 & 13.0 & 0.17 \\
\hline 1344 & CIV & 2.0 & 15.0 & 0.20 \\
\hline 1371 & O vi ${ }^{*}$ & 1.7 & 10.0 & 0.14 \\
\hline 1401 & Si IV & 1.7 & 9.4 & 0.12 \\
\hline 1410 & Si II & 2.2 & 12.0 & 0.16 \\
\hline 1425 & OVI & 1.5 & 8.2 & 0.11 \\
\hline 1486 & N IV & $\leq 1.0$ & $\leq 4.4$ & $\leq 0.057$ \\
\hline 1550 & $\mathrm{CIV}^{*}$ & abs & & \\
\hline 1640 & He II & 16.9 & 67.6 & 0.88 \\
\hline 1663 & O III] & $\leq 0.3$ & $\leq 1.1$ & $\leq 0.014$ \\
\hline 1750 & N III] & 3.7 & 12.3 & 0.16 \\
\hline 1762 & C II & $\leq 0.3$ & $\leq 1.1$ & $\leq 0.014$ \\
\hline 1776 & Fe II & 1.2 & 4.0 & 0.052 \\
\hline 1883 & Si III] & 0.4: & 1.5: & 0.020: \\
\hline 1892 & Si III] & $0.5:$ & 1.9: & $0.027:$ \\
\hline 1909 & C III] & 7.7 & 30.8 & 0.401 \\
\hline 2070 & OVI & 10.3 & 93.7 & 1.31 \\
\hline 2424 & [Ne IV] & 1.8 & 6.07 & 0.085 \\
\hline 2733 & He II & 1.2 & 1.25 & 0.018 \\
\hline 2836 & O III & 5.5 & 4.68 & 0.065 \\
\hline 3047 & O III & 5.3 & 3.29 & 0.046 \\
\hline 3132 & O III & 39.8 & 21.9 & 0.306 \\
\hline 3170 & $\mathrm{C}$ II? & 10.9 & 5.8 & 0.081 \\
\hline 3186 & Si II & 3.9 & 2.05 & 0.029 \\
\hline 3203 & He II & 6.4 & 3.23 & 0.045 \\
\hline
\end{tabular}

(1) I(line) measured - all intensity units $10^{-14} \mathrm{erg} \mathrm{cm}^{-2} \mathrm{~s}^{-1}$;

(2) I(line) extinction corrected - all intensity units $10^{-12} \mathrm{erg} \mathrm{cm}^{-2} \mathrm{~s}^{-1}$;

(3) $I($ line $) / \mathrm{I}(\mathrm{H} \beta)$ after correction for diaphragm size and extinction.

* These stellar lines show P Cygni profiles. The O v absorption has an equivalent width of $4.5 \AA$ and the C IV of $0.82 \AA$.

the measurements were made with respect to $\mathrm{H} \beta$, we have multiplied by the observed $\mathrm{H} \beta$. In this way no extinction corrections need to be applied and the measurements can be directly compared. If double weight is given to the strong O III line at $\lambda 3133 \AA$, a factor of 3.0 must be applied to the IUE measurements to bring into agreement with the ground based measurements. This is a reasonable number since since the infrared SWS measurements, made with a somewhat larger diaphragm, required an increase of 2.3.

The diaphragm size can also be found by comparing He II lines observed by the IUE with the total He II $\lambda 4686 \AA$, assuming the theoretical ratios given by Hummer \& Storey (1987) for a temperature of $8500 \mathrm{~K}$. This leads to a correction factor of 2.8 from the $\lambda 1640 \AA$ line and a factor of 3.5 from the less certain $\lambda 3202 \AA$ line. We have chosen to use the factor 2.8 for the SWP wavelength region and a factor 3.0 for the LWP region. The uncertainty of these factors is $10 \%-20 \%$; they have been used to calculate the ratio $I(\lambda) / I(\mathrm{H} \beta)$ given in the column labeled (3) in Table 6. 


\section{Chemical composition of the nebulae}

The method of analysis is the same as used in the papers cited in the introduction. First the electron density and temperature as function of the ionization potential are determined. Then the ionic abundances are determined, using density and temperature appropriate for the ion under consideration, together with Eq. (1). Then the element abundances are found for those elements in which a sufficient number of ions abundances have been derived.

\subsection{Electron density}

The ions used to determine $N_{\mathrm{e}}$ are listed in the first column of Table 7 (for NGC 40) and Table 8 (for NGC 6153). The ionization potential required to reach that ionization stage, and the wavelengths of the lines used, are given in Cols. 2 and 3 of the tables. Note that the wavelength units are $\AA$ when 4 ciphers are given and microns when 3 ciphers are shown. The observed ratio of the lines is given in the fourth column; the corresponding $N_{\mathrm{e}}$ is given in the fifth column. The temperature used is discussed in the following section, but is unimportant since these line ratios are essentially determined by the density.

There is no indication that the electron density varies with ionization potential in a systematic way in either of the nebulae. As already pointed out by Liu et al. (2001) the [O III] lines always give a lower density than the other lines. Ignoring these lines, the electron density appears to be about $2000 \mathrm{~cm}^{-3}$ for NGC 40 and about $4000 \mathrm{~cm}^{-3}$ for NGC 6153. The scatter is higher for NGC 40. It is interesting to compare this value of the density with the rms density found from the $\mathrm{H} \beta$ line. This depends on the distance of the nebula which isn't accurately known, and on the angular size of the nebula. For this calculation we shall use a distance of $0.8 \mathrm{kpc}$ for NGC 40 and $1.2 \mathrm{kpc}$ for NGC 6153. A sphere of radius of $19^{\prime \prime}$ will represent NGC 40 and $12^{\prime \prime}$ will represent NGC 6153. These are rather uncertain values. The $\mathrm{H} \beta$ flux has been given above and the electron temperature will be discussed below. We obtain the uncertain rms values of $1500 \mathrm{~cm}^{-3}$ for NGC 40 and $2600 \mathrm{~cm}^{-3}$ for NGC 6153. In spite of the uncertainties involved in these calculations (the uncertain distance and the assumption of a spherical homogeneous nebula), there is a similarity of these values to the forbidden line densities. This probably indicates that inhomogenieties do not play a dominant role in determining the density. We will use the forbidden line densities in further discussion of the abundances, i.e. $2000 \mathrm{~cm}^{-3}$ for NGC 40 and about $4000 \mathrm{~cm}^{-3}$ for NGC 6153 .

\subsection{Electron temperature}

A number of ions have lines originating from energy levels far enough apart that their ratio is sensitive to the electron temperature. These are listed in Tables 9 and 10, which are arranged similarly to the previous tables. The electron temperature for both nebulae remains roughly constant as a function of ionization potential at a similar rather low value. This is not always the case. Sometimes the higher stages of ionization are formed in higher temperature regions. This is not the case for these
Table 7. Electron density indicators in NGC 40.

\begin{tabular}{lcccc}
\hline \hline Ion & $\begin{array}{c}\text { Ioniz. } \\
\text { pot. }(\mathrm{eV})\end{array}$ & $\begin{array}{c}\text { Lines } \\
\text { used }\end{array}$ & $\begin{array}{c}\text { Observed } \\
\text { ratio }\end{array}$ & $\begin{array}{c}N_{\mathrm{e}} \\
\left(\mathrm{cm}^{-3}\right)\end{array}$ \\
\hline$[\mathrm{S}$ II $]$ & 10.4 & $6731 / 6716$ & 1.39 & 2100 \\
{$[\mathrm{O}$ II $]$} & 13.6 & $3626 / 3729$ & 1.3 & 1400 \\
{$\left[\mathrm{~N}_{\text {II }}\right]$} & 14.5 & $6584 / 122$ & 140. & 2000 \\
{$[\mathrm{~S} \mathrm{III}]$} & 23.3 & $33.5 / 18.7$ & 0.76 & 1100 \\
{$[\mathrm{Cl}$ III $]$} & 23.8 & $5538 / 5518$ & $1.3:$ & 4500 \\
{$[\mathrm{C}$ III $]$} & 24.4 & $1907 / 1909$ & 1.27 & 4000 \\
{$[\mathrm{O}$ III $]$} & 35.1 & $52 / 88$ & 1.7 & 800 \\
\hline
\end{tabular}

Table 8. Electron density indicators in NGC 6153.

\begin{tabular}{lcccc}
\hline \hline Ion & $\begin{array}{c}\text { Ioniz. } \\
\text { pot. }(\mathrm{eV})\end{array}$ & $\begin{array}{c}\text { Lines } \\
\text { used }\end{array}$ & $\begin{array}{c}\text { Observed } \\
\text { ratio }\end{array}$ & $\begin{array}{c}N_{\mathrm{e}} \\
\left(\mathrm{cm}^{-3}\right)\end{array}$ \\
\hline$[\mathrm{S} \mathrm{II}]$ & 10.4 & $6731 / 6716$ & 1.66 & 4100 \\
{$[\mathrm{O}$ II $]$} & 13.6 & $3626 / 3729$ & 1.92 & 3500 \\
{$[\mathrm{~S} \mathrm{III}]$} & 23.3 & $33.5 / 18.7$ & 0.28 & 4500 \\
{$[\mathrm{Cl}$ III $]$} & 23.8 & $5538 / 5518$ & 1.27 & 4500 \\
{$[\mathrm{O} \mathrm{III}]$} & 35.1 & $52 / 88$ & 3.61 & 2000 \\
{$[\mathrm{Ar}$ IV $]$} & 40.7 & $4711 / 4740$ & 1.08 & 2800 \\
{$[\mathrm{Ne}$ III $]$} & 41.0 & $15.5 / 36.0$ & 12.3 & 3400 \\
\hline
\end{tabular}

Table 9. Electron temperature indicators in NGC 40.

\begin{tabular}{lcccc}
\hline \hline Ion & $\begin{array}{c}\text { Ioniz. } \\
\text { pot. }(\mathrm{eV})\end{array}$ & $\begin{array}{c}\text { Lines } \\
\text { used }\end{array}$ & $\begin{array}{c}\text { Observed } \\
\text { ratio }\end{array}$ & $\begin{array}{c}T_{\mathrm{e}} \\
(\mathrm{K})\end{array}$ \\
\hline$[\mathrm{N}$ II $]$ & 14.5 & $5755 / 6584$ & 0.0078 & 7500 \\
{$[\mathrm{~S} \mathrm{III}]$} & 23.3 & $6312 / 18.7$ & 0.0145 & 7800 \\
{$[\mathrm{Ar}$ III $]$} & 27.6 & $7136 / 8.99$ & 0.488 & 7000 \\
{$[\mathrm{O} \mathrm{III}]$} & 35.1 & $4363 / 5007$ & 0.0062 & 10500 \\
{$[\mathrm{O}$ III $]$} & 35.1 & $5007 / 52$ & 2.15 & 8000 \\
{$[\mathrm{Ne}$ III $]$} & 41.0 & $3868 / 15.5$ & 0.295 & 8400 \\
\hline
\end{tabular}

Table 10. Electron temperature indicators in NGC 6153.

\begin{tabular}{lcccc}
\hline \hline Ion & $\begin{array}{c}\text { Ioniz. } \\
\text { pot. }(\mathrm{eV})\end{array}$ & $\begin{array}{c}\text { Lines } \\
\text { used }\end{array}$ & $\begin{array}{c}\text { Observed } \\
\text { ratio }\end{array}$ & $\begin{array}{c}T_{\mathrm{e}} \\
(\mathrm{K})\end{array}$ \\
\hline$[\mathrm{N}$ II $]$ & 14.5 & $5755 / 6584$ & 0.017 & 9600 \\
{$[\mathrm{~S} \mathrm{III}]$} & 23.3 & $6312 / 18.7$ & 0.016 & 8000 \\
{$[\mathrm{Ar}$ III $]$} & 27.6 & $7136 / 8.99$ & 0.342 & $\leq 7000$ \\
{$[\mathrm{O}$ III $]$} & 35.1 & $4363 / 5007$ & 0.0047 & 9030 \\
{$[\mathrm{O}$ III $]$} & 35.1 & $5007 / 52$ & 3.6 & 7800 \\
{$[\mathrm{O}$ III $]$} & 35.1 & $1663 / 5007$ & $\leq 0.00159$ & $\leq 7900$ \\
{$[\mathrm{Ne}$ III $]$} & 41.0 & $3868 / 15.5$ & 0.290 & 8200 \\
\hline
\end{tabular}

nebulae, but also not for several other nebulae with low electron temperatures.

Notice that for both nebulae the temperature determined from the O III line ratio $\lambda 4363 \AA / \lambda 5007 \AA$ is higher than that determined from ratios of other lines of the same ion. It is not clear if this is a measurement error or whether there is a physical explanation. Both nebulae have a similar electron temperature and it is difficult to judge the weight to be given to different ions. We have chosen to give both nebulae an average temperature of $8200 \mathrm{~K}$. This is similar to the temperature found by Clegg et al. (1983) for NGC $40\left(T_{\mathrm{e}}=8300 \mathrm{~K}\right)$. It is somewhat 
lower than that used by Liu et al. (2000) for NGC 6153 who did not use the ISO SWS measurements.

\subsection{Ionic and element abundances}

The ionic abundances have been determined using the following equation:

$\frac{N_{\text {ion }}}{N_{\mathrm{p}}}=\frac{I_{\text {ion }}}{I_{\mathrm{H}_{\beta}}} N_{\mathrm{e}} \frac{\lambda_{\mathrm{ul}}}{\lambda_{\mathrm{H}_{\beta}}} \frac{\alpha_{\mathrm{H}_{\beta}}}{A_{\mathrm{ul}}}\left(\frac{N_{\mathrm{u}}}{N_{\mathrm{ion}}}\right)^{-1}$

where $I_{\mathrm{ion}} / I_{\mathrm{H}_{\beta}}$ is the measured intensity of the ionic line compared to $\mathrm{H} \beta, N_{\mathrm{p}}$ is the density of ionized hydrogen, $\lambda_{\mathrm{ul}}$ is the wavelength of this line, $\lambda_{\mathrm{H}_{\beta}}$ is the wavelength of $\mathrm{H} \beta, \alpha_{\mathrm{H}_{\beta}}$ is the effective recombination coefficient for $\mathrm{H} \beta, A_{\mathrm{ul}}$ is the Einstein spontaneous transition rate for the line, and $N_{\mathrm{u}} / N_{\mathrm{ion}}$ is the ratio of the population of the level from which the line originates to the total population of the ion. This ratio has been determined using a five level atom.

\subsubsection{NGC 40}

The results are given in Table 11, where the first column lists the ion concerned, and the second column the line used for the abundance determination. The third column gives the intensity of the line used relative to $\mathrm{H} \beta=100$. The fourth column gives the ionic abundances, and the fifth column gives the Ionization Correction Factor (ICF). This has been determined empirically. Notice that the ICF is unity (except for $\mathrm{P}$ and $\mathrm{Fe}$ ) and the element abundances, given in the last column, are probably well determined. Only the abundance of $\mathrm{C}^{+3}$ is uncertain because the presence of such a high ionization stage is unexpected. Clegg et al. (1983) have already noted this, and have investigated the possibility that the line is formed by resonance scattering of starlight. They conclude that there may be a contribution of radiation produced in the inner part of the nebula and scattered from the region observed. For this reason we do not include the $\mathrm{C}^{+3}$ ion in the total carbon abundance.

The abundances in NGC 40 are in general very similar to solar abundances. This is shown in Table 13 where it can be seen that the abundances of oxygen, neon and argon agree almost exactly with those of the sun. Sulfur is lower than solar, but in most nebulae it is lower and it is likely that the solar abundance is anomalous. Even nitrogen is only slightly higher than solar. Carbon is the only element with a substantially higher abundance: a factor of five above solar. This large abundance has already been partially seen by Clegg et al. (1983) (who didn't recognize it as an overabundance). Iron is clearly depleted with respect to the solar value. This is seen in all nebulae where iron has been measured, and is presumably due to depletion onto dust grains.

\subsubsection{NGC 6153}

The results are given in Table 12 which has the same format as the previous table.

The abundances in NGC 6153 are quite different from NGC 40. Carbon and oxygen are about a factor of two higher
Table 11. Ionic concentrations and chemical abundances in NGC 40. Wavelength in Angstrom for all values of $\lambda$ above 1000, otherwise in $\mu \mathrm{m}$.

\begin{tabular}{lccccc}
\hline \hline Ion & $\lambda$ & Intens./H $\beta$ & $N_{\text {ion }} / N_{\mathrm{p}}$ & ICF & $N_{\text {el. } /} / N_{\mathrm{p}}$ \\
\hline $\mathrm{He}^{+}$ & 5875 & 6.5 & 0.044 & & \\
$\mathrm{He}^{+}$ & 4471 & 2.43 & 0.048 & & \\
$\mathrm{C}^{+}$ & 2325 & 75. & $8.54(-4)$ & & \\
$\mathrm{C}^{++}$ & 1909 & 66. & $9.33(-4)$ & & \\
$\mathrm{C}^{+3}$ & 1548 & $\leq 25$. & $\leq 7.4(-4)$ & 1 & $1.9(-3)$ \\
$\mathrm{N}^{+}$ & 6584 & 283. & $1.01(-4)$ & & \\
$\mathrm{N}^{+}$ & 122 & 1.85 & $8.6(-5)$ & & \\
$\mathrm{N}^{++}$ & 57 & 9.83 & $3.46(-5)$ & 1 & $1.3(-4)$ \\
$\mathrm{O}^{+}$ & 3727 & 380. & $5.0(-4)$ & & \\
$\mathrm{O}^{++}$ & 5007 & 25. & $1.9(-5)$ & & \\
$\mathrm{O}^{++}$ & 52 & 11.6 & $3.0(-5)$ & & \\
$\mathrm{O}^{+3}$ & 25,8 & $\leq 0.14$ & $\leq 5.2(-8)$ & 1 & $5.3(-4)$ \\
$\mathrm{Ne}^{+}$ & 12.8 & 78.6 & $1.38(-4)$ & & \\
$\mathrm{Ne}^{++}$ & 15.5 & 1.16 & $8.4(-7)$ & & \\
$\mathrm{Ne}^{++}$ & 3869 & 0.34 & $8.3(-7)$ & 1 & $1.4(-4)$ \\
$\mathrm{S}^{+}$ & 6731 & 14.5 & $1.48(-6)$ & & \\
$\mathrm{S}^{++}$ & 18.7 & 34.2 & $4.09(-6)$ & & \\
$\mathrm{S}^{++}$ & 6312 & 0.5 & $2.8(-6)$ & 1 & $5.6(-6)$ \\
$\mathrm{Ar}^{+}$ & 6.99 & 17.4 & $2.39(-6)$ & & \\
$\mathrm{Ar}^{++}$ & 8.99 & 9.48 & $7.2(-7)$ & 1 & $3.4(-6)$ \\
$\mathrm{Ar}^{++}$ & 7136 & 4.6 & $3.8(-6)$ & & \\
$\mathrm{Cl}^{++}$ & 5538 & 0.27 & $7.5(-8)$ & & \\
$\mathrm{Cl}^{++}$ & 5518 & 0.20 & $5.9(-8)$ & & \\
$\mathrm{P}^{++}$ & 17.9 & 2.08 & $1.2(-7)$ & $2.0:$ & $2.4(-7):$ \\
$\mathrm{Fe}^{+}$ & 25.9 & $0.23:$ & $6.2(-8)$ & & \\
$\mathrm{Fe}^{++}$ & 22.93 & 0.93 & $3.4(-7)$ & $1.5:$ & $6.1(-7):$ \\
$\mathrm{Si}^{+}$ & 34.8 & 4.05 & $2.0(-6)$ & & \\
$\mathrm{Si}^{++}$ & 1892 & 2.4 & $4.9(-6)$ & 1 & $7.0(-6)$ \\
\hline & & & & & \\
\hline
\end{tabular}

Intensities given with respect to $\mathrm{H} \beta=100$.

than solar, while neon and argon are almost a factor of three higher. Sulfur is about solar which, as discussed above, is already a factor of two or three higher than in most nebulae. Nitrogen is a factor of five higher than solar, but this also true of many other nebulae. It appears that NGC 6153 was formed from material which originally had a higher abundance than solar, and only nitrogen and helium have increased by nucleosynthesis in the course of evolution.

\section{Comparison with other abundance determinations}

Tables 13 and 14 shows a comparison of our abundances with the most important determinations in the past $20 \mathrm{yrs}$. There is marginal agreement, usually to within a factor of two. A comparison is also made with the solar abundance (Anders \& Grevesse 1989; Grevesse \& Sauval 1998; Allende Prieto et al. 2001), which has been commented on above.

The helium abundance has been derived using the theoretical work of Benjamin et al. (1999). For recombination of singly ionized helium, most weight is given to the $\lambda 5875 \AA$ line , because the theoretical determination of this line is the most reliable. The abundance of helium in NGC 40 is a lower limit since 
Table 12. Ionic concentrations and chemical abundances in NGC 6153. Wavelength in Angstrom for all values of $\lambda$ above 1000, otherwise in $\mu \mathrm{m}$

\begin{tabular}{|c|c|c|c|c|c|}
\hline Ion & $\overline{\bar{\lambda}}$ & "Intens./H $\beta$ & $\overline{\bar{N} N_{\text {ion }} / N_{\mathrm{p}}}$ & $\overline{\mathrm{ICF}}$ & 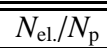 \\
\hline $\mathrm{He}^{+}$ & 5875 & 19.6 & 0.131 & & \\
\hline $\mathrm{He}^{+}$ & 4471 & 6.51 & 0.128 & & \\
\hline $\mathrm{He}^{++}$ & 4686 & 12.8 & 0.010 & 1 & 0.14 \\
\hline $\mathrm{C}^{++}$ & 1909 & 40.1 & $5.67(-4)$ & 1.2 & $6.8(-4)$ \\
\hline $\mathrm{N}^{+}$ & 6584 & 53.5 & $1.90(-5)$ & & \\
\hline $\mathrm{N}^{+}$ & 122 & $\leq 0.18$ & $\leq 2(-5)$ & & \\
\hline $\mathrm{N}^{++}$ & 1750 & $16 .:$ & $5.9(-4)$ & & \\
\hline $\mathrm{N}^{++}$ & 57 & 72.1 & $4.42(-4)$ & 1.1 & $4.8(-4)$ \\
\hline $\mathrm{O}^{+}$ & 3727 & 28.8 & $4.40(-5)$ & & \\
\hline $\mathrm{O}^{++}$ & 5007 & 908. & $6.87(-4)$ & & \\
\hline $\mathrm{O}^{++}$ & 52 & 251. & $1.0(-3)$ & & \\
\hline $\mathrm{O}^{+3}$ & 25.8 & 97.3 & $3.7(-5)$ & 1 & $8.3(-4)$ \\
\hline $\mathrm{Ne}^{+}$ & 12.8 & 11.5 & $1.95(-5)$ & & \\
\hline $\mathrm{Ne}^{++}$ & 15.5 & 311. & $2.26(-4)$ & & \\
\hline $\mathrm{Ne}^{++}$ & 3869 & 90.2 & $2.20(-4)$ & & \\
\hline $\mathrm{Ne}^{+3}$ & 2425 & 8.5 & $6.10(-5)$ & 1 & $3.1(-4)$ \\
\hline $\mathrm{S}^{+}$ & 6731 & 5.86 & $6.0(-7)$ & & \\
\hline $\mathrm{S}^{++}$ & 18.7 & 86.3 & $1.04(-5)$ & & \\
\hline $\mathrm{S}^{++}$ & 6312 & 1.42 & $7.78(-6)$ & & \\
\hline $\mathrm{S}^{+3}$ & 10.5 & 267. & $8.54(-6)$ & 1.05 & $1.9(-5)$ \\
\hline $\mathrm{Ar}^{+}$ & 6.99 & 11.4 & $1.57(-6)$ & & \\
\hline $\mathrm{Ar}^{++}$ & 8.99 & 53.4 & $6.09(-6)$ & & \\
\hline $\mathrm{Ar}^{++}$ & 7136 & 21.9 & $3.39(-6)$ & & \\
\hline $\mathrm{Ar}^{+3}$ & 4740 & 2.35 & $1.48(-6)$ & 1 & $8.5(-6)$ \\
\hline $\mathrm{Fe}^{++}$ & 22.9 & 0.42 & $1.55(-7)$ & 2: & $3.1(-7)$ \\
\hline $\mathrm{Cl}^{++}$ & 5538 & 0.744 & $2.1(-7)$ & & \\
\hline $\mathrm{Cl}^{++}$ & 5518 & 0.581 & $1.7(-7)$ & & \\
\hline $\mathrm{Cl}^{+3}$ & 11.8 & 4.05 & $2.7(-7)$ & 1.2 & $5.6(-7)$ \\
\hline $\mathrm{Na}^{++}$ & 7.32 & 1.77 & $1.6(-6)$ & & \\
\hline $\mathrm{Si}^{+}$ & 34.8 & 2.8 & $2.0(-6)$ & & \\
\hline $\mathrm{Si}^{++}$ & 1892 & 2.7: & $5.5(-6)$ & 1.3 & $9.8(-6)$ \\
\hline
\end{tabular}

the ionization in this nebula is so low that much neutral helium may exist.

\subsection{Errors}

It is difficult to determine the errors in the abundance determination. The reason for this is the following. The error can occur at several stages in the determination. An error can occur in the intensity determination and this can be specified: it is probably less than $30 \%$ and may be lower for the stronger lines. An error may occur in correcting for the extinction, either because the extinction is incorrect or the average reddening law is not applicable. We have tried to minimize this possibility by making use of known atomic constants to relate the various parts of the spectrum. Thus the ratio of the infrared spectrum to the visible spectrum is fixed by the ratio of $\mathrm{Br} \alpha$ to $\mathrm{H} \beta$ which is an atomic constant.

A further error is introduced by the correction for unseen stages of ionization. This varies with the element, but is usually small because very many ionization stages are observed. Thus for neon all but neutral neon is observed, so that the error is negligible. This is also true for sulfur, argon, oxygen and
Table 13. Comparison of abundances in NGC 40.

\begin{tabular}{lrrrr}
\hline \hline Elem. & Present & Clegg(1) & $\mathrm{AC}(2)$ & Solar(3) \\
\hline $\mathrm{He}$ & $\geq 0.046$ & $\geq 0.044$ & $\geq 0.05$ & 0.098 \\
$\mathrm{C}(-4)$ & 19 & 10 & & 3.3 \\
$\mathrm{~N}(-4)$ & 1.3 & 2.4 & 0.98 & 0.84 \\
$\mathrm{O}(-4)$ & 5.3 & 8.4 & 6.0 & 4.9 \\
$\mathrm{~S}(-6)$ & 5.6 & 3.9 & 4.1 & 19 \\
$\mathrm{Ar}(-6)$ & 3.4 & & & 2.5 \\
$\mathrm{Ne}(-4)$ & 1.4 & & & 1.2 \\
$\mathrm{Fe}(-7)$ & 6.1 & & & 320 \\
\hline
\end{tabular}

(1) Clegg et al. (1983); (2) Aller \& Czyzak (1979); (3) Solar: Anders \& Grevesse (1989); Allende Prieto et al. (2001); Grevesse \& Sauval (1998).

Table 14. Comparison of abundances in NGC 6153.

\begin{tabular}{lrrrr}
\hline \hline Elem. & Present & Liu(1) & PDM(2) & Solar(3) \\
\hline $\mathrm{He}$ & 0.14 & 0.14 & 0.14 & 0.098 \\
$\mathrm{C}(-4)$ & 6.8 & 2.8 & 8.0 & 3.3 \\
$\mathrm{~N}(-4)$ & 4.8 & 2.3 & 20 & 0.84 \\
$\mathrm{O}(-4)$ & 8.3 & 5.0 & 10 & 4.9 \\
$\mathrm{~S}(-5)$ & 1.9 & 1.6 & 3.7 & 1.9 \\
$\mathrm{Ar}(-6)$ & 8.5 & 2.7 & 10 & 2.5 \\
$\mathrm{Ne}(-4)$ & 3.1 & 1.7 & 2.5 & 1.2 \\
$\mathrm{Cl}(-7)$ & 5.6 & 4.2 & & 3.0 \\
$\mathrm{Fe}(-7)$ & $3.1:$ & & & 320 \\
\hline
\end{tabular}

(1) Liu et al. (2000); (2) Pottasch et al. (1986); (3) Solar: Anders \& Grevesse (1989); Allende Prieto et al. (2001); Grevesse \& Sauval (1998).

nitrogen where the higher stages of ionization which are not observed contribute very little to the abundance.

There is also an error due to an incorrect determination of the electron temperature. This is very small for ions represented by infrared lines, so that the abundances of neon, argon and sulfur will not be affected. The abundance of carbon is more sensitive to the temperature however. This is one of the reasons that we obtain a higher carbon abundance in NGC 6153 than Liu et al. (2000). It is not a reason for the higher carbon abundance found in NGC 40, since the electron temperature we found is almost the same as used by Clegg et al. (1983).

\subsection{Recombination line abundances}

Liu et al. (2000) have discussed the recombination line abundances in NGC 6153 for several ions of carbon, nitrogen, oxygen and neon using their careful measurements of very weak lines from this nebula. These abundances are a factor of 3 to 5 higher than we have obtained from the collisional lines. (These authors find a factor of 10 difference because they use lower collisional line abundances). The explanation for this difference is not clear. Originally it was suggested that temperature inhomogeneities caused a too high temperature to be found; a lower temperature would increase the collisional line abundances. But now that collisional line abundances can be determined from low lying levels which are insensitive to the temperature (the infrared lines) it is clear that some other 
explanation must be found. Liu et al. (2001) have examined several models based on the assumption that there exist hydrogen-poor regions in the nebula which have a low electron temperature so that only recombination line radiation is emitted. None of these models is entirely satisfactory however.

In NGC 6153 the $\mathrm{C}^{++}$recombination line $\lambda 4267 \AA$ yields an abundance $\mathrm{C}^{++} / \mathrm{H}$ of $2.2 \times 10^{-3}$. We have also obtained an upper limit for the $\mathrm{C}^{++}$recombination line $\lambda 1761 \AA$ which yields a much lower upper limit to the abundance: $\mathrm{C}^{++} / \mathrm{H} \leq 2.6 \times 10^{-4}$. These abundances are obtained using the effective recombination coefficients given by Davey et al. (2000) for their Case B, which refers to the case in which the nebula is optically thick for the resonance transition. If the nebula is completely thin (Case A) for the resonance transition the abundance from the $\lambda 1761 \AA$ would be increased by a factor of about 5.5 , while that obtained from the $\lambda 4267 \AA$ would essentially be unchanged. In this way agreement may almost be achieved. We regard it as unlikely however that the nebula is completely thin for the resonance line $(\lambda 1334 \AA)$.

Consider now the same lines in NGC 40. In this case the $\lambda 1761 \AA$ line is much stronger and yields a Case B abundance $\mathrm{C}^{++} / \mathrm{H}=1.5 \times 10^{-3}$. This is a factor 4 higher than obtained from the $\lambda 4267 \AA$ line. Here we cannot invoke the optically thin case, because this would only increase the difference. The only possibility of obtaining agreement is if the measurement of the $\lambda 4267 \AA$ by Clegg et al.(1983) is too small by a factor 4 . This is unlikely since the spectra given by these authors shows the line very clearly and its intensity relative to $\mathrm{H} \beta$ does not have an error of more than $30 \%$. The possibility that the $\lambda 1761 \AA$ line is misidentified is also unlikely because an additional $\mathrm{C}^{++}$line is seen with an intensity which gives the same higher abundance as the $\lambda 1761 \AA$ line. This is the $\mathrm{C}^{++}$line at $\lambda 2836$ which is difficult to measure in many nebulae because it is blended with a Bowen O III line. This is not the case in NGC 40 because almost no O III is present. This would argue that there is some physical process which is not yet well enough understood to predict the observed $\mathrm{C}^{++}$recombination line ratios. This makes it uncertain that the correct $\mathrm{C}^{++}$abundance can be determined from its recombination lines.

\section{The central star}

\subsection{NGC 40}

As mentioned in Sect. 1, this nebula is excited by a WC8 central star. Using the visual magnitude of 11.55 (obtained from the HST measurements of Ciardullo et al. 1999) and the $\mathrm{H} \beta$ flux given above, the hydrogen Zanstra temperature $\left(T_{\mathrm{z}}(\mathrm{H})\right)$ is about $35000 \mathrm{~K}$. No doubly ionized helium is seen in the nebula. This is consistent with the above temperature. The "Stoy" or Energy Balance temperature can also be found from the above data. The value of the ratio of "forbidden line emission" (including all collisionally excited emission) to $\mathrm{H} \beta$ is 12.3 , which leads to an Energy Balance temperature $\left(T_{\mathrm{EB}}\right)$ of $41000 \mathrm{~K}$, assuming blackbody emission. A model atmosphere would give a slightly lower temperature (see Preite-Martinez \& Pottasch 1983). We will use a stellar temperature of $38000 \mathrm{~K}$ in the following discussion.
As already mentioned in the introduction, Bianchi \& Grewing (1987) obtain a temperature of $T=90000 \mathrm{~K}$ from the ultraviolet (IUE) continuum radiation. This evidence is very uncertain. Firstly, it is strongly dependent on the value of extinction used. Bianchi \& Grewing use a value of $E_{B-V}=0.50$. The value found here, $E_{B-V}=0.41$, reduces the continuum temperature to a value of about $T=60000$. Secondly, even small departures of the continuum from a black body can have an important effect on the derived temperature, especially because a limited wavelength region is being observed in a wavelength region where only a small percentage of the total flux is contained. Therefore there appears to be little reason to doubt that the temperature is about $38000 \mathrm{~K}$.

\subsection{NGC 6153}

The central star has an observed visual magnitude of 15.55 (Ciardullo et al. 1999). Corrected for extinction, $E_{B-V}=0.81$, this is a magnitude of 13.04, and leads to a hydrogen Zanstra temperature $T_{\mathrm{z}}(\mathrm{H})=74000 \mathrm{~K}$. The ionized helium Zanstra temperature is slightly higher $T_{\mathrm{z}}(\mathrm{HeII})=84000 \mathrm{~K}$. The value of the ratio of "forbidden line emission" (including all collisionally excited emission) to $\mathrm{H} \beta$ is 26.7 , which leads to an energy balance temperature $\left(T_{\mathrm{EB}}\right)$ of $80000 \mathrm{~K}$, which we will use as a reasonable to the temperature of the star.

\subsection{Radius and luminosity}

These quantities are dependent on the distance of the nebulae which is difficult to obtain accurately. For this discussion we will use the distances cited above: $d=0.8 \mathrm{kpc}$ (NGC 40) and $d=1.2 \mathrm{kpc}$ (NGC 6153). Similar values are cited in the literature especially as "individual distances". These values have an uncertainty which could be larger than $40 \%$. They lead to stellar radii $R / R_{\odot}=0.73$ (NGC 40) and $R / R_{\odot}=0.19$ (NGC 6153) and, using the above temperatures, stellar luminosities $L / L_{\odot}=720$ (NGC 40) and $L / L_{\odot}=950$ (NGC 6153).

It is also possible to obtain the stellar luminosity from the nebular $\mathrm{H} \beta$ luminosity, since there is a direct relationship between the number of ionizing photons and the number of $\mathrm{H} \beta$ photons, in the case in which the nebula absorbs all the ionizing photons emitted by the star. A mathematical formulation of this can be found in Pottasch \& Acker (1989). It yields the following luminosities: $L / L_{\odot}=760$ (NGC 40) and $L / L_{\odot}=860$ (NGC 6153). This is roughly the same found above and indicates that most of the ionizing photons are absorbed in the nebula. It does not give any information about the distance, since both formulations have the same distance dependance.

\section{Discussion and conclusions}

The rather low luminosity of the central star of both of these nebulae, although quite uncertain, leads to the expectation that the initial mass of the star was low so that only the first dredgeup (and possibly the third dredge-up) took place. This seems in general agreement with the abundance determinations. In NGC 40 the abundances are solar (with the exception of carbon). Even nitrogen is only slightly increased compared to the 
Table 15. Comparison of abundances in PN with WR central stars.

\begin{tabular}{lrrrr}
\hline \hline Elem. & NGC 40 & BD+303639 & NGC 5315 & Solar(3) \\
\hline $\mathrm{He}$ & $\geq 0.046$ & $\geq 0.0053$ & 0.124 & 0.098 \\
$\mathrm{C}(-4)$ & 19 & 7.3 & 4.4 & 3.3 \\
$\mathrm{~N}(-4)$ & 1.3 & 1.1 & 4.6 & 0.84 \\
$\mathrm{O}(-4)$ & 5.3 & 4.6 & 5.2 & 4.9 \\
$\mathrm{~S}(-6)$ & 5.6 & 6.4 & 12 & 21 \\
$\mathrm{Ar}(-6)$ & 3.4 & 5.2 & 4.6 & 2.5 \\
$\mathrm{Ne}(-6)$ & 1.4 & 1.9 & 1.6 & 1.2 \\
\hline
\end{tabular}

$\natural$ Abundances from Bernard-Salas et al. (2003).

$\dagger$ Abundances from Pottasch et al. (2002).

sun, indicating that only the first dredge-up took place. The large carbon abundance is puzzling. It could have some relation to the carbon rich WR central star which is beginning to lose some of its atmosphere to the nebula. However this has not been seen to this extent in other nebulae with WR central stars which we have investigated. This is illustrated in Table 15 which compares the abundances in NGC 40 with those in two other nebulae with Wolf-Rayet central stars: BD+303639 (WC9) and NGC 5315 (WC4).

From the table a large similarity of the abundances of oxygen, neon, argon and sulfur is seen. Nitrogen is considerably higher in NGC 5315 indicating that it is a somewhat higher mass star and a second dredge-up has occurred. Neither of these nebulae has such a high $\mathrm{C} / \mathrm{O}$ ratio as NGC 40. It is clear that the high carbon abundance is anomalous.

In NGC 6153 the abundances of neon, argon, oxygen, carbon, and chlorine are between a factor of 2 and 3 higher than solar. The sulfur abundance is equal to that of the sun, but is about a factor of 2 to 3 higher than in other planetary nebulae. The only other element measured, nitrogen, is about a factor 5 higher. It seems unlikely that this increase could have taken place in the course of the evolution of the star. It is more likely that the central star of this nebula originally was formed from material with an abundance 2 to 3 times higher than solar. Further, it appears to be a star of low enough mass that the third dredge-up could not take place.

The role of the intensity of the $\mathrm{C}^{++}$recombination line intensities is discussed. It appears difficult to explain the ratios of $\lambda 4267 \AA / \lambda 1761 \AA$ in both nebulae with the same recombination line theory. The theory may have to be reconsidered.

\section{References}

Allende Prieto, C., Lambert, D. L., \& Asplund, M. 2001, ApJ, 556, L63

Aller, L. H., \& Czyzak, S. J. 1979, Ap\&SS, 62, 397
Aller, L. H., Czyzak, S. J., Buerger, E. G., et al. 1972, ApJ, 172, 361

Anders, E., \& Grevesse, N. 1989, Geochem. Cosmo., 53, 197

Becker, R. H., White, R. L., \& Edwards, A. L. 1991, ApJS, 75, 1

Benjamin, R. A., Skillman, E. D., \& Smits, D. P. 1999, ApJ, 514, 307

Bernard Salas, J., Pottasch, S. R., Beintema, D. A., \& Wesselius, P. R. 2001, A\&A, 367, 949

Bernard-Salas, J., Pottasch, S. R., Wesselius, P. R., \& Feibelman, W. A. 2003, A\&A, 406, 165

Bianchi, L., \& Grewing, M. 1987, A\&A, 181, 85

Cahn, J. H., Kaler, J. B., \& Stanghellini, L. 1992, A\&AS, 94, 399

Carrasco, L., Serrano, A., \& Costero, R. 1983, RMxAA, 8, 187

Ciardullo, R., Bond, H. E., Sipior, M. S., et al. 1999, AJ, 118, 488

Clegg, R. E. S., Seaton, M. J., Peimbert, M., et al. 1983, MNRAS, 205,417

Condon, J. J., \& Kaplan, D. L. 1998, ApJS, 117, 361

Crowther, P. A., De Marco, O., \& Barlow, M. J. 1998, MNRAS, 296, 36

Davey, A. R., Storey, P. J., \& Kisielius, R. 2000, A\&AS, 142, 85

de Freitas Pacheco, J. A., Maciel, W. J., Costa, R. D. D., et al. 1991, A\&A, 250, 159

Feibelman, W. A. 1999, ApJ, 514, 296

Fluks, M. A., Plez, B., de Winter, D., et al. 1994, A\&AS, 105, 311

George, D., Kaftan-Kassim, M. A., \& Hartsuiker, A. P. 1974, A\&A, 35,219

Gregory, P. C., \& Condon, J. J. 1991, ApJS, 75, 1011

Gregory, P. C., Vavasour, J. D., Scott, W. K., et al., 1994, ApJS, 90, 173

Grevesse, N., \& Sauval, A. J. 1998, Space Sci. Rev., 85, 161

Griffith, M. R., Burke, B. F., \& Ekers, R. D. 1994, ApJS, 91, 111

Hippelein, H., \& Munch, G. 1981, A\&A, 95, 100

Hummer, D. G., \& Storey, P. J. 1987, MNRAS, 224, 801

Johansson, S. 1988, ApJ, 327, L85

Kingsburgh, R. L., \& Barlow, M. J. 1994, MNRAS, 271, 257

Liller, W. 1955, ApJ, 122, 240

Liu, X.-W., Barlow, M. J., Cohen, M., et al. 2001, MNRAS, 323, 343

Liu, X.-W., Storey, P. J., Barlow, M. J., et al. 2000, MNRAS, 312, 585

Meaburn, J., Lopez, J. A., Bryce, M., \& Mellema, G. 1996, A\&A, 307, 579

Pottasch, S. R., \& Acker, A. 1989, A\&A, 221, 123

Pottasch, S. R., Wesselius, P. R., Wu, C. C., et al. 1977, A\&A, 54, 435

Pottasch, S. R., Dennefeld, M., \& Mo, J.-E. 1986, A\&A, 155, 397

Pottasch, S. R., \& Beintema, D. A. 1999, A\&A, 347, 974

Pottasch, S. R., Beintema, D. A., \& Feibelman, W. A. 2000, A\&A, 363, 767

Pottasch, S. R., Beintema, D. A., Bernard Salas, J., \& Feibelman, W. A. 2001, A\&A, 380, 684

Pottasch, S. R., Preite-Martinez, A., Olnon, F. M., et al. 1986, A\&A, 161,363

Pottasch, S. R., Beintema, D. A., Bernard Salas, J., et al. 2002, A\&A 393, 285

Sabbadin, F., Cappellaro, E., Benetti, S., et al. 2000, A\&A, 355, 688

Preite-Martinez, A., \& Pottasch, S. R. 1983, A\&A, 126, 31

Seiber, W., Haslam, C. G. T., \& Salter, C. J. 1979, A\&A, 74, 361

Smith, L. F., \& Aller, L. H. 1969, ApJ, 157, 1245 